\begin{tabular}{lc}
\hline & Sharif University of Technology \\
SCIENTIA & Transactions B: Mechanical Engineering \\
IRAN ICA & www.scientiairanica.com \\
\hline
\end{tabular}

Research Note

\title{
A novel method for maximum lift prediction in high-lift configurations
}

\author{
M. Pasandideh Fard* and S.A. Sahaf \\ Department of Mechanical Engineering, Ferdowsi University of Mashhad, Mashhad, Iran.
}

Received 28 April 2014; received in revised form 19 December 2014; accepted 10 August 2015

\section{KEYWORDS}

High lift device; Aircraft design; Maximum lift; Reynolds average Navier stokes.

\begin{abstract}
Many decisions in the preliminary design steps of an aircraft are very hard to take, due to a lot of unknown variables at this stage. These early decisions can be made more reliable by testing different configurations by numerical methods, repetitively. Therefore, it is very important to have a rapid, reliable and particularly easy to implement numerical tool. One of the most important steps in aerodynamic configuration development is the design and sizing of high lift devices. The main criterion for this design is the lift increment that a particular configuration can produce. Therefore, it is very important to adequately estimate the maximum lift coefficient for a flapped wing at highly deflected flap configurations. This paper tries to introduce a novel numerical-empirical method for estimation of the lift generation capability of a specific high lift device configuration. However, drag production estimation is not in the scope of this paper. In this method, the linear portion of the lift curve is derived numerically, while the curved near stall region is estimated through empirical methods. The results are compared with some experimental data to show the method validity.
\end{abstract}

(C) 2016 Sharif University of Technology. All rights reserved.

\section{Introduction}

The design and analysis of aerospace vehicles and structures are becoming more and more reliant on Computational Fluid Dynamics (CFD). The engineers increasingly use numerical methods, like IBL (Interactive Boundary Layer) and RANS (Reynolds Average Navier Stokes) methods, in their designs and studies. The main reason for this approach is CFD advantages over traditional wind tunnel experiments. Some of these advantages are; lower cost, a faster process and the ability to repeat a test easily. Moreover, wind tunnel testing has a severe limitations. Most wind tunnels cannot operate at flight Reynolds numbers, and, unfortunately, scale effects are not yet completely

*. Corresponding author. Mobile: +98 9155179018;

E-mail addresses: fard_m@um.ac.ir (M. Pasandideh Fard); sahaf.mech@gmail.com (S.A. Sahaf) understood. Therefore, using wind tunnel data alone to accomplish an aerodynamic shape design can introduce a significant amount of uncertainty. On the other hand, CFD has not proven itself reliable enough for sole use in this regard. Currently, CFD is generally considered reliable only for flight regimes near cruise conditions, when there is little or no separated flow present and when high-lift devices are not deployed. However, the main challenge here is to predict the flow at high angles of attack, where the separation is very likely to happen. Aircraft takeoff and landing are good examples of such a challenge, where the flow is highly deflected and the use of multi section flap systems inserts multiple boundary layers and transitions to the flow. In most flow analyses, the drag estimation is the main challenge and the lift prediction is more convenient. However, flow analysis during takeoff and landing is so complicated that most times, predicting maximum lift becomes a CFD challenge. Hence, in the 
analysis of high-lift flow, in the early design steps, there are two simultaneous issues that should be taken care of. Firstly, the analysis should be performed easily and quickly, and secondly, the method must be consistent with high lift flow necessity. These two parameters (cost efficiency and precision) are the case even in automatic numerical high lift flow optimizations [1]. There are some similar efforts existing in the literature and some are introduced in this article as examples.

Schuster and Birkelbaw [2] are two pioneers in the field of flow simulation over a multi-element airfoil, using a structured grid RANS code. Their test case was a GA(W)-1 two-element airfoil. This work was done so long ago that the grid was very coarse (only 11721 or 2457 total points), and the algebraic BL turbulence model was employed. With such a coarse grid, the results were not good at all, but it was a good start.

Fritz [3] solved RANS equations to compute the flow over a $2.6 \%$-gap flap on an NLR-7301 two-element airfoil. The grid was structured and a $k-\epsilon$ model was selected for the turbulence modeling. Predicted lift levels were reasonable, but surface pressure on the upper surface of the flap did not agree well with experiments. Among previous affairs in the literature of high lift flow analysis, Godin et al. [4] did very well. They used a grid with over 180,000 points to compute both the $2.6 \%$ - and $1.3 \%$-gap cases of a NLR-7301 two-element airfoil, using a structured RANS code. Their experiments were so accurate that they managed to note that, in the $2.6 \%$-gap case, this gap contracts in the order of $0.2 \%$, in practice. Therefore, they used the measured value of $2.4 \%$ of $\mathrm{C}$ rather than the nominal value. They also made the transition point fixed, based on experimental results. They selected Spalart-Almaras and Menter's SST $k-\omega$ to model the turbulence effects. Their results were very precise for both pressure coefficients and maximum lift coefficients. However, it should be noted that when it comes to aircraft design, we need to simulate the flow hundreds of times for specific cases when we may not have any experimental results, including the transition point, deflections of the gap surfaces, laminar bubble extensions, and etc. Moreover, we should not make the simulations too heavy and time consuming (very high resolution grids) which make the aircraft design process practically stop. This makes the process a bit more challenging.

As a more practical and industrial example, EUROLIFT II is a project developed in the German Aerospace Center (DLR) to adequately understand CFD capabilities in predicting high lift [5]. In this project, researchers presented similar test cases for five different European institutions and gathered their results. The main objective of this project was assessment of CFD capabilities in predicting dependence of the high-lift performance on the Reynolds number, and to further improve understanding of the high-lift associated vortex phenomena, especially the nacelle strake mechanism. Although they were using 3-D RANS codes and the results were developed and admitted by five different European institutions, independently, their results suffer from lack of accuracy.

There are also some affairs in the literature whose focus is on the optimization of high-lift devices and to set the best location for the flaps and slats based on the main airfoil position [6,7]. In these kinds of affair, the researcher is interested to know the trend of the lift and drag variation with manipulation of high lift device items. Hence, the precision of value estimation is of less interest; that is why, in most investigations, unstructured grids are used and high cost solvers are usually avoided. Many of these affairs also use rapid IBL methods for faster results [8].

As implied in this section, the interest in highlift computations has been considerable over the last decade, and many CFD papers have been written about them. Most papers (in the order of 100) deal with 2-D computations, and about one-fourth as many references deal with $3-\mathrm{D}$ multi-element computations. Recent years have also seen an increase in the rate of publications in this area [9]. Many of these efforts were diverse and usually uncompleted, until the first AIAA high lift prediction workshop in June 2010, which was really a step forward. This workshop illustrated the significance of high lift prediction and invited numerical aero-dynamists to elaborate on this challenge. AbdolHamid published his 3-D CFD results on the first AIAA high lift prediction workshop [10]. These high precision $3-\mathrm{D}$ calculations (with more than 30 million grids) were successful, and managed to accurately estimate the lift and drag coefficients. These results are extremely different to the previous 2-D comparative results, which were not accurate at all. As a good example in this regard, Klausmeyer and Lin [11] performed a comparative study of CFD capability in lift and drag prediction in $2-\mathrm{D}$. The results were really poor and problematic.

These comparative studies show that, firstly, CFD analyses are more challenging in 2-D, and higher accuracy is expected in 3-D calculations. Moreover, high lift prediction is not a simple attached flow lift prediction, and involves a great deal of aerodynamic knowledge to accurately simulate the phenomenon. Different aerodynamic incidents occur in this phenomenon which increase simulation difficulties.

To sum up, it should be said that high lift prediction is a really big challenge. This challenge becomes more complicated, considering the requirements of the process of aircraft design. The design process, during its first stages, is a trial and error process. Therefore, it is desired to develop a method which is very easy and fast to implement and estimates lift at high lift flow 
rapidly and also accurately. This is exactly what will be discussed in this paper.

As mentioned earlier, this paper endeavors to present a novel numerical-empirical method that can predict the lift performance of a flapped airfoil accurately, rapidly and much more easily. It should be noted that this method can also easily estimate lift increments due to flap deployment for any flapped wing configuration and for any flap deflection angle. This estimation can be very helpful in the early phases of design and sizing of a high lift device.

It should be noted that, most of the time, implementing new methods helps to solve traditional problems $[12,13]$.

\section{Maximum lift, numerical calculation strategy for high lift configurations}

The main objective of High Lift Devices (H.L.D) numerical calculations is to find the lift increments due to flap deployment and the value of maximum lift coefficient. In other words, it is very important for an H.L.D. designer to sketch the lift curve diagram $(\mathrm{Cl}$ AOA) prior to any other diagram. The strategy of this paper for performing H.L.D calculations is to calculate the lift coefficient for lower angles of attack (linear portion of $\mathrm{Cl}$-AOA diagram), and then extrapolate the results for higher angles of attack, based on a semi-empirical method innovatively derived from [14]. This strategy helps to limit calculations to the lower angles of attack, where the lift curve is linear and the numerical simulation is much easier (say, less than 8 deg.). According to Roskam's method [14], which is a very widespread aircraft design method, there is a reasonable relation between $\Delta C l$ and $\Delta C l_{\max }$. These parameters are $C l$ increments, due to flap deflections at 0 degrees, and maximum lift angle of attack, respectively. The relation between $\Delta C l$ and $\Delta C l_{\text {max }}$ is shown in Figure 1, based on [14]. As illustrated in this figure, the ratio of $\Delta C l_{\max } / \Delta C l$, shown by parameter " $K$ ", is a function of flap type and flap chord ratio (i.e., $C_{f} / C$ ).

It seems that parameter " $K$ " refers to extending the separated regions because of flap deployment. This is the reason why " $K$ " approaches " 1 " when the flap chord goes to zero. Moreover, " $K$ " goes to unity when the flap system benefits from one or two slots. These slots avoid the abrupt propagations of separation zones. According to the definition of parameter " $K$ ", one can calculate the lift increment at a 0 degree angle of attack (i.e. $\Delta C l$ ) of a wing or airfoil, numerically, and predict the stall region based on parameter " $K$ ". This procedure is shown in Figure 2. As illustrated in Figure 2, the $\mathrm{Cl}$-AOA diagram for a clean wing or airfoil must firstly be sketched. (There is no severe problem or difficulty in estimating the aerodynamic coefficients for a clean wing.) For the flapped airfoil,

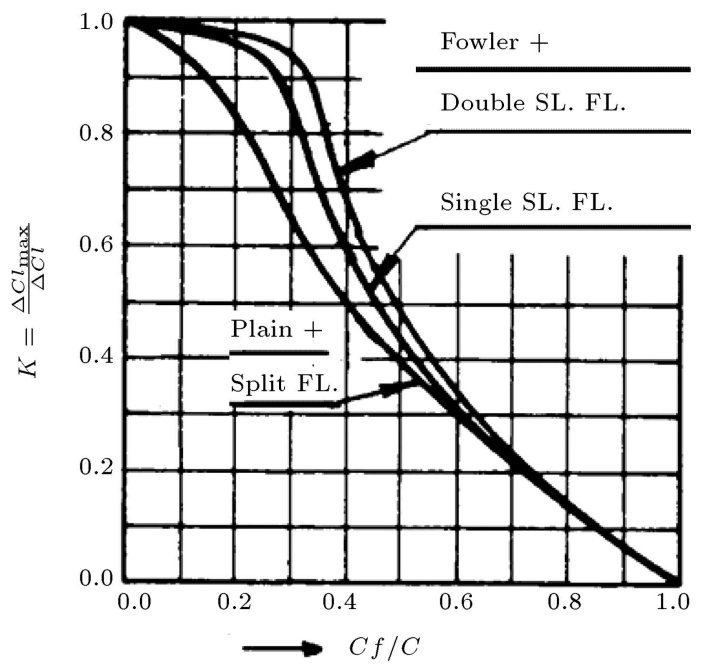

Figure 1. $C l$ increments relation $(K)$ according to the flap chord ratio $\left(C_{f} / C\right)$ taken from [14].

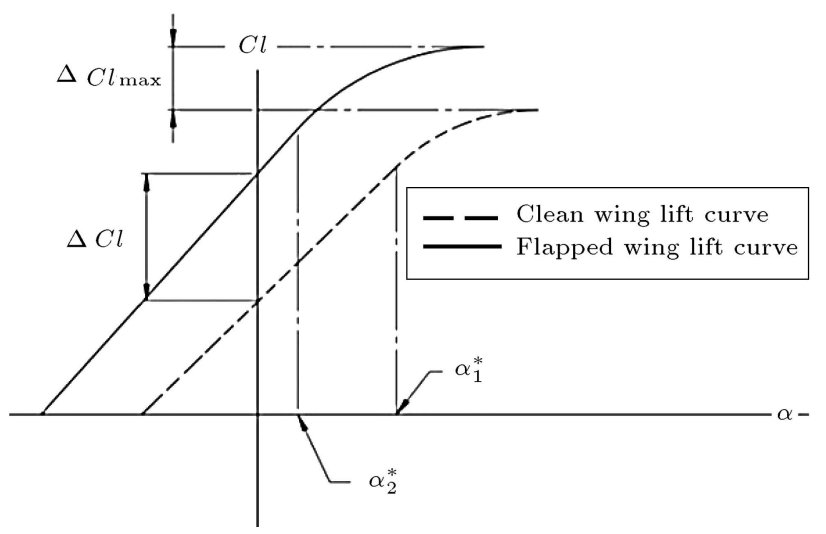

Figure 2. Definition and illustration of relevant parameters.

the linear portion of the $C l$-AOA diagram can be derived using numerical methods. So, the value of $\Delta \mathrm{Cl}$ can be estimated. Consequently, the value of $\Delta C l_{\max }$ is derived using parameter " $K$ ", as discussed before. As the value of $C l_{\max }$, for a clean wing, is known already, the value of the maximum lift coefficient for a flapped wing can be calculated using the values, $\Delta C l_{\max }$ and $C l_{\text {max }_{\text {clean wing }} \text {. }}$

$$
C l_{\text {max }_{\text {flapped wing }}}=C l_{\text {max }_{\text {clean wing }}}+\Delta C l_{\max } \text {. }
$$

This procedure, which seems to be very effective, especially in preliminary design steps, is completely novel and efficient. The main benefit of this method is skipping numerical calculations at higher angles of attack and limiting the study domain to the linear portion of the $\mathrm{Cl}-\alpha$ diagram.

\section{Method validation}

To validate this method, any experimental lift curve can be used. For instance, experimental results of [15] 


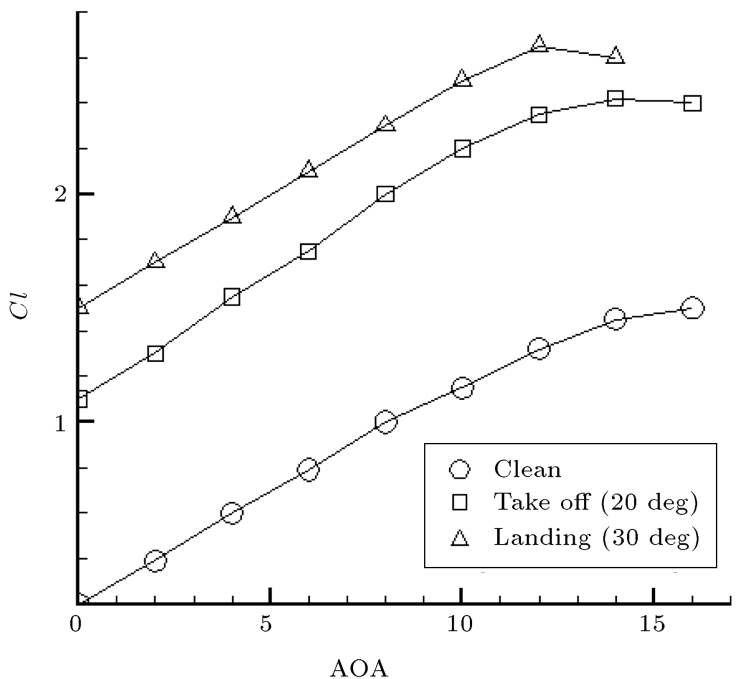

Figure 3. $\mathrm{Cl}$-AOA for 23012 clean airfoil according to experiments [15].

Table 1. NACA-23012 airfoil experimental lift data.

\begin{tabular}{lccc}
\hline \multicolumn{1}{c}{ Flap position } & $\boldsymbol{C l}(\mathbf{A O A}=\mathbf{0})$ & $\boldsymbol{C l}_{\max }$ & $\boldsymbol{\alpha}^{*}$ \\
\hline Clean & 0.2 & 1.5 & 8 \\
Flap down $(20 \mathrm{deg})$ & 1.1 & 2.42 & 10 \\
Flap down $(30 \mathrm{deg})$ & 1.5 & 2.65 & 8 \\
\hline
\end{tabular}

were used to validate the procedure above. The $C l-\alpha$ diagram for a NACA 23012 airfoil in a clean position, was derived experimentally from [15]. Moreover, the same curves were generated for the flap down case, at different flap angles, according to experimental data. These graphs are illustrated in Figure 3. The lift curve properties are shown in Table 1.

Let us assume that the lift coefficients in the near stall regions (i.e. high angles of attack), for the flap down cases in Figure 3, are not known. It is desired to estimate maximum $\mathrm{Cl}$ and the near stall portion of the lift curves for flap down cases (Takeoff-Landing), based on the experimental results of the linear portions. To do so, Tables 1 and 2 are developed. These tables contain all the requirements for estimating the lift curve diagram for higher angles of attack and near stall regions.

Based on Table 1, and the procedure discussed earlier, the data of Table 2 can be derived.

As illustrated in Table 2, the level of errors is very low, and the results are extremely precise and accurate, even in the stall region, which is very hard to simulate.

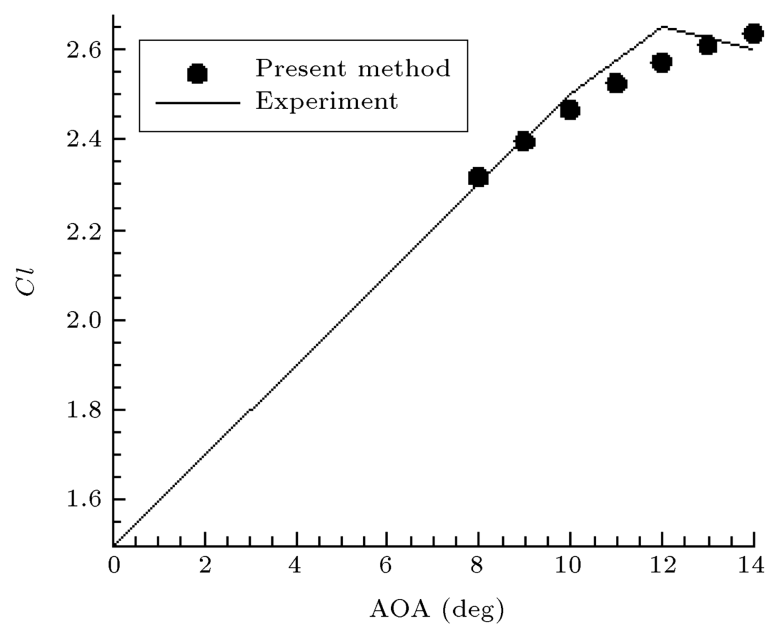

Figure 4. Comparison of semi-empirical method with experimental results.

According to the presented method, the lift curve $(\mathrm{Cl}-$ $\alpha$ ) diagram can be sketched, using the linear portion of the experimental lift curve and the values of $C l_{\max }$ and $\alpha^{*}$ of Tables 1 and 2 . The near stall portion of the lift curve is assumed to be a 2nd degree polynomial curve. The result of this validation is shown in Figure 4.

$$
\begin{aligned}
& C l=A \alpha^{2}+B \alpha+C \\
& \text { If } \alpha=\alpha^{*} \text { then } \quad d C l / d \alpha=C_{l_{\alpha}}, \\
& d C l / d \alpha=2 A \alpha+B \\
& \text { If } \alpha=\alpha_{c l_{\max }} \text { then } d C l / d \alpha=0, \\
& C=C l_{\max }-A \alpha_{C l \max }^{2}-B \alpha_{C l_{\max }} .
\end{aligned}
$$

It worth mentioning that this high accuracy was expected, since, as mentioned in the literature [16], the lift curve diagram for the flapped airfoil is a translated copy of the lift diagram for the original clean airfoil. Sometimes a rotation is also added to the translation vector. Hence, if one knows the variation of $\alpha^{*}$, one can estimate the variation of maximum lift angle of attack $\left(\alpha_{C l \text { max }}\right)$, accordingly.

The data of Table 2 and Figure 4 show that this method is valid. So, all calculations can be confined to the linear range of angles of attack and the lift coefficient for higher angles of attack can be calculated through empirical relations. This will reduce the CPU time dramatically, while keeping the error band limited.

Table 2. $\mathrm{Cl}_{\max }$ evaluation using the introduced semi empirical model and comparing the result with the experiments.

\begin{tabular}{ccccccc}
\hline Flap position & $\boldsymbol{\Delta} \boldsymbol{C l}$ & $\boldsymbol{K}$ (see Figure 1) & $\boldsymbol{\Delta} \boldsymbol{C} \boldsymbol{l}_{\max }$ & $\boldsymbol{C l}_{\text {max }_{\text {Present work }}}$ & $\boldsymbol{C l}_{\text {max }_{\exp }}$ & Error (\%) \\
\hline Flap down (20 deg) & 0.9 & 0.96 & 0.864 & 2.364 & 2.42 & 2.31 \\
Flap down (30 deg) & 1.3 & 0.96 & 1.248 & 2.748 & 2.65 & 3.7 \\
\hline
\end{tabular}




\section{Numerical calculation for modelling the linear range of lift curve}

Although the problem has since been made much easier, it has not yet been solved. This part of the article tries to provide the reader with a suitable numerical set-up which can be adequately used to model the H.L.D. lift performance in a linear range of angles of attack.

\subsection{Grid generation}

The grid generation for modeling the H.L.D aerodynamics is very important. This phase itself can be a complete, separate project. See, for example, Cao and Kusunose [17] and Rogers et al. [18]. Therefore, a complete study was performed to find the best solution for grid generation. In this study, the effect of cell shape, grid resolution and different grid parameters of boundary layers are studied.

\subsubsection{Grid cell shape effect}

The very first parameter to be analyzed is the grid cell shape. Two different grids were developed in this study. The first grid is a structured quad-map grid, while the second is an unstructured tri-grid. Rumyantsevin and Silantiev [19] strongly recommend using structured grids for H.L.D. numerical calculations and claim that very accurate results were obtained using a structured grid. A structured grid was, therefore, firstly used in this article. The grid size has been suggested to be so fine that the $Y^{+}$remains in the order of unity [10, 11, 19]. Smaller $Y^{+}$proved to have negligible improvement in the lift prediction results [10] (however, this further refinement may be useful in drag prediction). This grid is shown in Figure 5. As illustrated in this figure, the domain has been divided into many sub domains to generate a structured map grid. After refining the structured grid to make it independent from grid size effects, the results were compared with experimental data. The experimental data are captured from [15] for a NACA 23012-2h flapped airfoil at 30 degree flap deflection angle. The results showed very good consis-

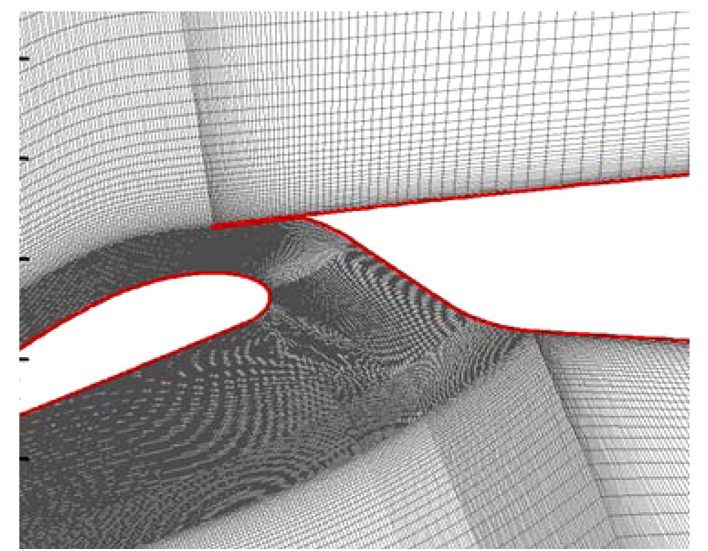

Figure 5. Structured grid for a slotted flapped airfoil.

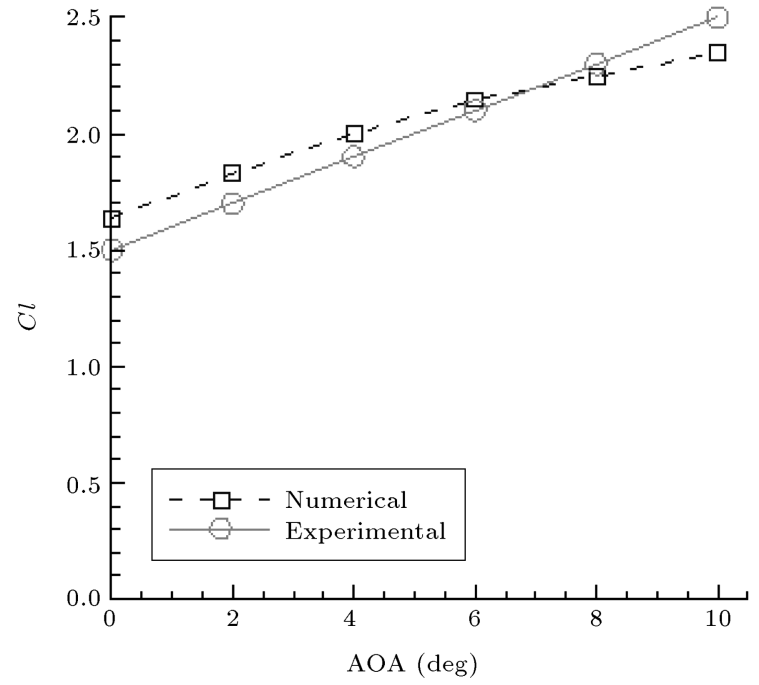

Figure 6. $\mathrm{Cl}$-AOA diagram for the numerical results of using structured grid and its comparison with experimental results (NACA 23012-2h flap deflection $=$ 30) [13].

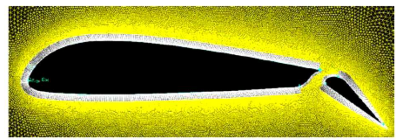

$\Delta X_{\text {surf }}=0.002$

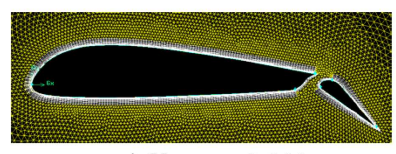

$\Delta X_{\text {surf }}=0.020$

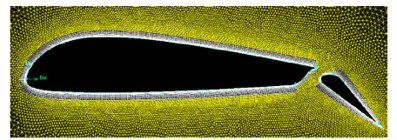

$\Delta X_{\text {surf }}=0.005$

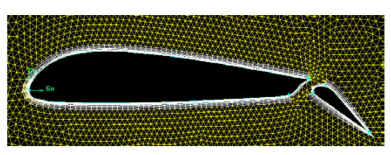

$\Delta X_{\text {surf }}=0.010$
Figure 7. Study of grid interval size.

tency with experimental data (see Figure 6). Despite accurate results, this grid is very time consuming to be generated, and, therefore, is not suitable for early design steps. Hence, a complete study was performed to develop a simple unstructured grid.

\subsubsection{Grid interval size}

A complete study was performed on the grid interval size, for the unstructured grid. The results and the grids are introduced next. Figure 7 shows different grids generated based on different surface interval sizes. As shown in this figure, the smaller surface grid interval sizes result in finer area grids. The question is; how far do we need to lower the surface grid interval size and what is the optimum value for this parameter?

The results of lift coefficient estimation for different interval sizes are compared in Figure 8. As seen in this figure, the lift coefficient results are very close to each other, especially for interval sizes smaller than 0.01 . So, the interval size of grid spacing can be chosen as 0.01 .

\subsubsection{Effect of using boundary layer grid}

As Figure 9 shows, different numbers of rows for the boundary layer grid in the vicinity of the walls 


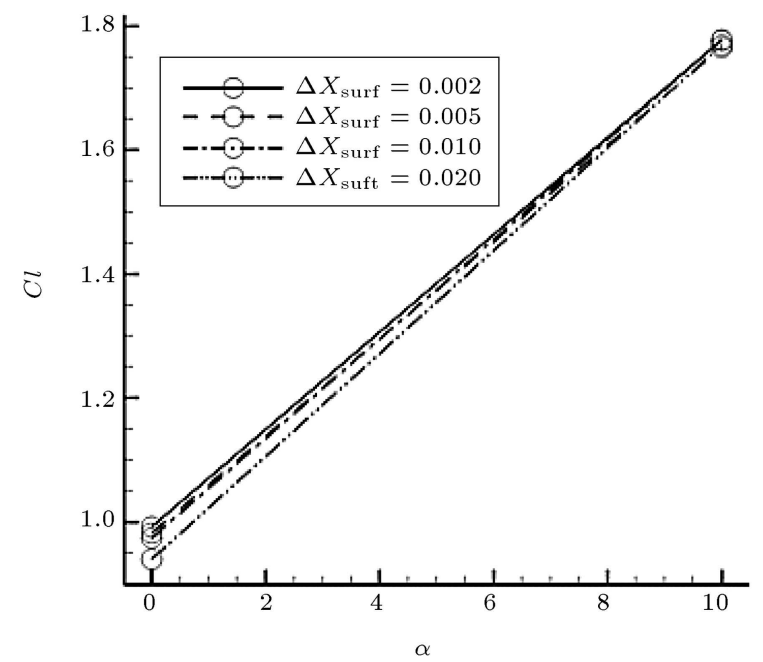

Figure 8. Effect of grid size on lift coefficient.

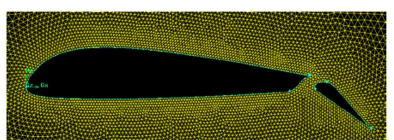

$N_{L}=0$

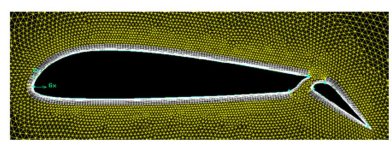

$N_{L}=10$

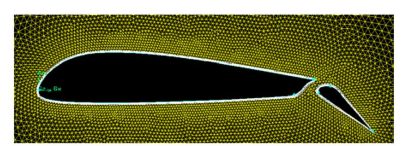

$N_{L}=5$

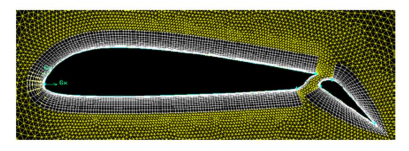

$N_{L}=15$
Figure 9. Effect of using different numbers of boundary layer grid rows on the flapped airfoil.

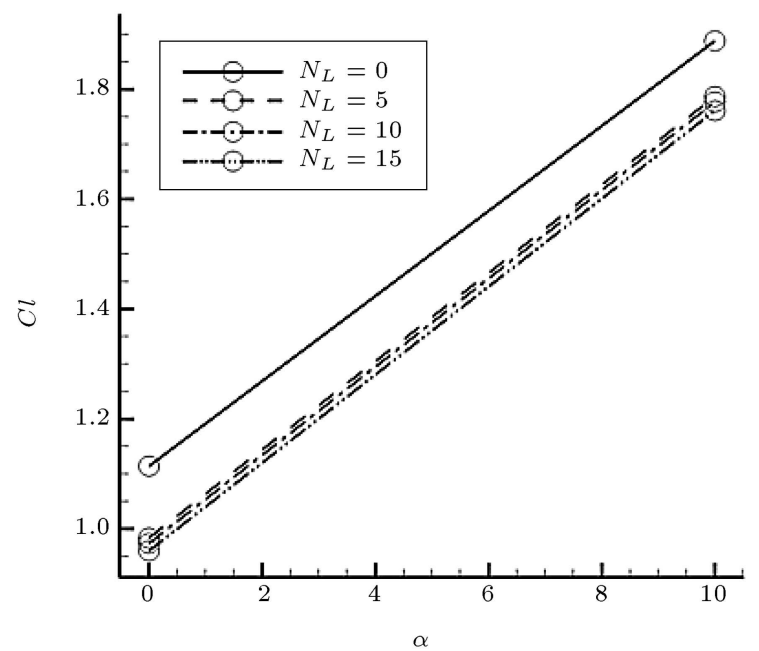

Figure 10. Effect of using boundary layer grid on lift coefficient.

were considered. Each of these grids was used in the numerical set-up separately and the lift estimation was performed. The results of lift estimations are illustrated in Figure 10. As Figure 10 suggests, the effect of using a boundary layer grid is very small (up to $7 \%$ ) and it was clearly decided not to use this type of mesh in the numerical set-up. The probable reason
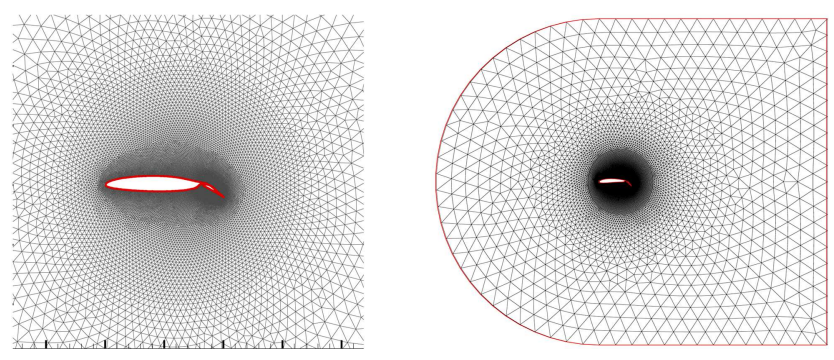

Figure 11. The overall view of the suggested unstructured tri-grid.

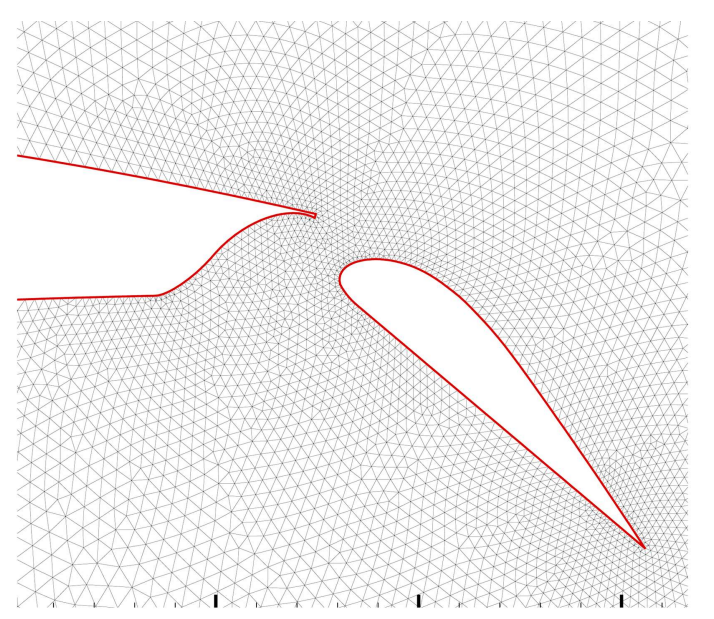

Figure 12. Detailed view of the suggested unstructured tri-grid.

is mesh inconsistency. As an unstructured tri-grid is used in the domain, using a few layers of a structured boundary layer quad grid cannot create any special improvement in results. However, it should be noted that these few layers of structured quad grids have improved the results and lowered the over estimation of numerical results in the linear range. But. the influence is not that much and it seems better not to add further complications to the grid generation process. Therefore, the number of boundary layer rows is set to be equal to zero.

\subsubsection{Final unstructured tri-grid}

Based on the grid study performed previously, the final unstructured tri-grid can be developed. Figures 11 and 12 show the final grid and its domain.

Having optimized the unstructured tri-grid, we must undertake a comparative study to find the level of changes in the results due to the use of an unstructured grid. Figure 13 suggests that there is only a very small difference between these two grids after performing optimization for an unstructured grid. The error band limit, for both structured and unstructured grids, is compared in Table 3. As shown in this table, the computational errors for both types of grid are extremely close to each other. Therefore, an unstructured grid can clearly be a better option, because of its simplicity. 
Table 3. Comparison of error bands between the best results of structured and unstructured grids.

\begin{tabular}{ccccc}
\hline \multirow{2}{*}{ AOA } & $\begin{array}{c}\boldsymbol{C l} \\
\text { (Unstructured grid) }\end{array}$ & $\begin{array}{c}\text { Error\% } \\
\text { (Unstructured grid) }\end{array}$ & $\begin{array}{c}\boldsymbol{C l} \\
\text { (Structured }\end{array}$ & $\begin{array}{c}\text { Errid) } \\
\text { (Structured grid) }\end{array}$ \\
\hline 0 & 1.6355 & 9.033333 & 1.6214 & 8.093333333 \\
2 & 1.83 & 7.647059 & 1.8101 & 6.476470588 \\
4 & 2 & 5.263158 & 1.979 & 4.157894737 \\
6 & 2.146 & 2.190476 & 2.15 & \\
8 & 2.246 & -2.34783 & 2.2407 & -2.57826087 \\
10 & 2.348 & -6.08 & 2.37 & -5.2 \\
\hline
\end{tabular}

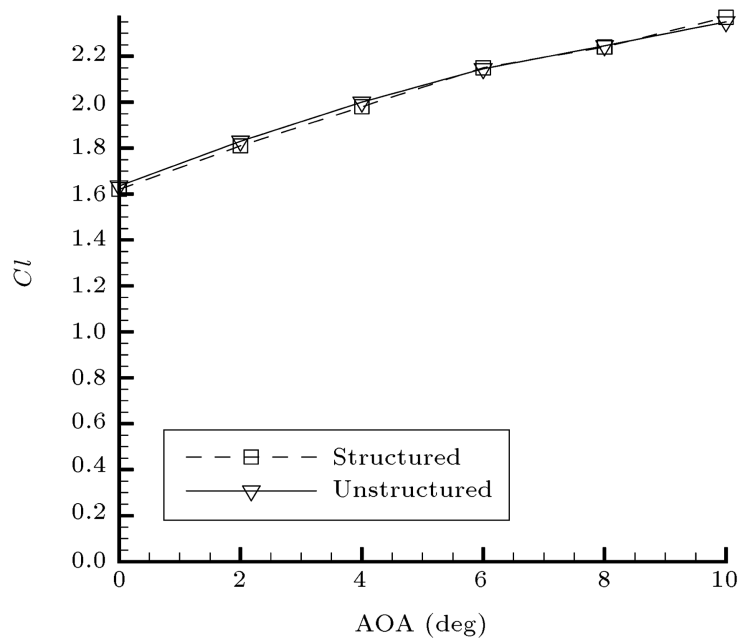

Figure 13. Comparison of two grids results.

Unstructured mesh can be as good as structured mesh in lift prediction.

Therefore, this paper uses the optimized tri-grid for estimating lift coefficients in the linear range.

\subsection{Turbulence modeling}

One of the most important parameters that should be adequately set up for deriving reasonable results in modeling H.L.D. is the turbulence model. Especially at higher angles of flap deployment (landing), it is very important to have a good estimation of the birth and dissipation of vortices. These vortices will definitely affect the lift and drag values for the flapped wing. Modern flap systems benefit from different methods of boundary layer triggering and energizing methods, which lead to a more stable flow with smaller separated zones. However, all these interactions in the boundary layer need a suitable turbulence model to be modeled appropriately. Turbulence models are generally categorized into two main categories. The first category, called the "high Reynolds" turbulence model, is that which simulates the turbulence effect by modeling turbulence behavior in the regions far from the wall, while using wall functions for simulating turbulence effects in the vicinity of the wall. The other group is composed of turbulence models that undertake turbulence modeling for the entire fluid domain. The former group is called the "low Reynolds" turbulence model. Based on what has been mentioned for the flow characteristics, and different interactions inside the boundary layer in high lift aerodynamics, it seems that the low Reynolds turbulence model group is the right choice. Some of the most important turbulence models in this group are the $K-\omega s s t$ and RSM models. Moreover, LES and DES are also capable of modeling interactions inside the boundary layer, very effectively. The DNS method is a new, precise method well beyond the capabilities of the present computers. Although it was decided to use low Reynolds turbulence models, theoretically, it is strongly recommended not to put the Sp-Al model aside. This model is a simple oneequation, high Reynolds model, which proved to have superb performance in aerodynamic applications. This article tries $K-\omega s s t$ and RSM as low Reynolds models, and $\mathrm{Sp}-\mathrm{Al}$ as a high Reynolds model to predict the lift performance of a flapped airfoil. These models will be evaluated based on deviation of their results from existing experimental results.

\subsection{Boundary conditions}

Boundary conditions are the physics identity of the problem. So, they should be properly set, in order to solve the right problem. In this article, the results will be compared with experimental data. In other words it is proposed to simulate the flow over a flapped airfoil in a wind tunnel with the specifications mentioned in [15]. So, the fluid domain boundaries must be set as walls, in order to input the wall interference effects into the solution domain. Moreover the flow will not be ideal and definitely has a grade of turbulence intensity, mentioned in [15], which is $6 \%$. This turbulent intensity greatly affects the lift coefficients. It should be noted that after the validation step, when the design process starts, no wall entity should be put around the domain (free stream condition will be imposed) and the turbulence intensity must be selected according to the practical takeoff/landing conditions.

The effective Reynolds number $\left(\mathrm{Re}_{e}\right)$ of the tests was set approximately 3,500,000, which corresponded to a velocity of $35.76 \mathrm{~m} / \mathrm{s}$. This Reynolds number was chosen based on test conditions mentioned in [15]. 


\section{Results and discussion}

Based on the numerical set-up introduced previously, the lift performance of the flapped airfoil NACA 23012$2 \mathrm{~h}$ was investigated. It should be noted that this flapped airfoil is introduced in [15], where all its geometrical details and wind tunnel results are available. As mentioned before, three different turbulence models were used and their results were compared to each other. Note that in these numerical efforts, an unstructured grid was used. Figure 14 shows the linear portion of the lift curve, according to three different turbulence models.

Table 4 compares the results of two grid types, with different turbulence models, with the experimental values.

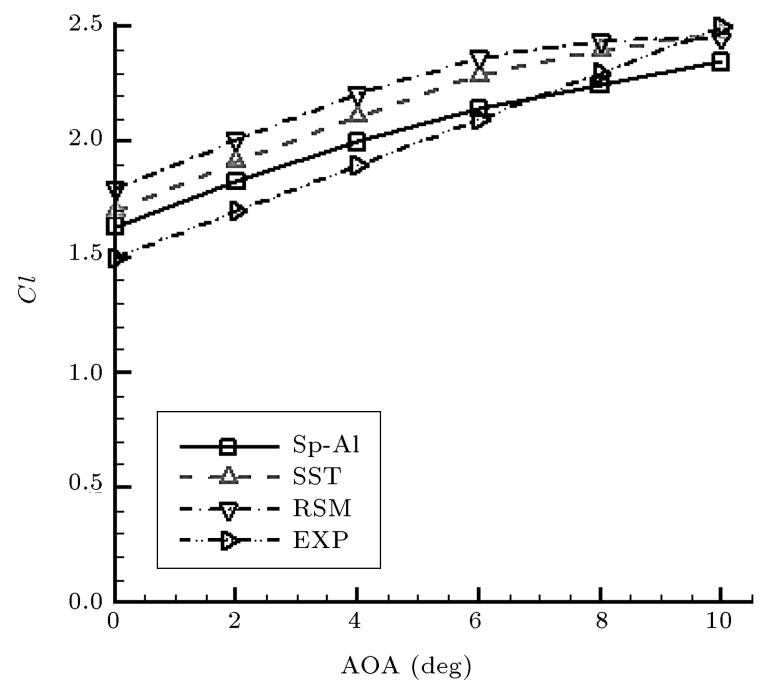

Figure 14. Effect of turbulence model. Both high Reynolds and low Reynolds turbulence models are included. (Test case is NACA 23012-2h flapped airfoil at 30 degree flap deflection angle (Wenzinger \& Harris, 1993)[15]).

\section{Extending the linear range results to higher angles of attack}

The results for the linear portion of the lift curve can be easily extended for the near stall region based on the semi-empirical method presented in this article. The results of such an effort are illustrated in Figure 15. These results are compared to the experimental results, and the error bands are shown in Table 5 .

As Table 5 and Figure 15 show, there are big positive errors for small angles of attack, while, for high angles of attack, the errors are small and negative. In other words, the numerical calculations overestimate the lift for lower angles of attack and underestimate lift for higher ones. This is while they produce reasonable results in 6 to 8 degree angles. To explain this trend, we should pay attention to flow separation. In the lower angles of attack, the flow separates at the trailing edge due to the flap deployment and high flow curvature. In

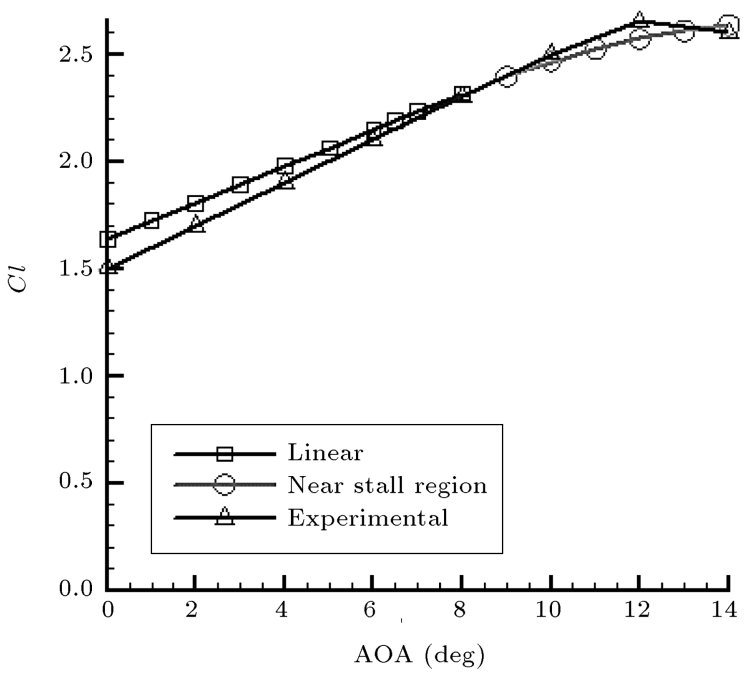

Figure 15. Extending the linear results for the near-stall region.

Table 4. Structured and unstructured grids $C l$ values and comparison of the results with experiments.

\begin{tabular}{|c|c|c|c|c|c|}
\hline \multirow{2}{*}{ Grid type } & \multicolumn{3}{|c|}{ Unstructured grid } & \multirow{2}{*}{$\begin{array}{c}\text { Structured grid } \\
\mathrm{Sp}-\mathrm{Al}\end{array}$} & \multirow{2}{*}{$\begin{array}{c}\text { Experimental } \\
\text { data }\end{array}$} \\
\hline & SP-Al & SST & RSM & & \\
\hline 0 & 1.6355 & 1.916 & 2.01 & 1.6214 & 1.5 \\
\hline 2 & 1.83 & 2.11 & 2.21 & 1.8101 & 1.7 \\
\hline 4 & 2 & 2.291 & 2.367 & 1.979 & 1.9 \\
\hline 6 & 2.146 & 2.4 & 2.44 & 2.15 & 2.1 \\
\hline 8 & 2.246 & 2.475 & 2.45 & 2.2407 & 2.3 \\
\hline 10 & 2.348 & 1.916 & 2.01 & 2.37 & 2.5 \\
\hline
\end{tabular}

Table 5. Error bands (\%) for different angles of attack for both linear and near stall regions.

\begin{tabular}{lcccccccc}
\hline Zone/AOA & 0 & 2 & 4 & 6 & 8 & 10 & 12 & 14 \\
Linear & 9.03 & 6.22 & 3.99 & 2.19 & 0.71 & - & - & - \\
Near-stall & - & - & - & - & - & -1.39 & -2.96 & 1.36 \\
\hline
\end{tabular}




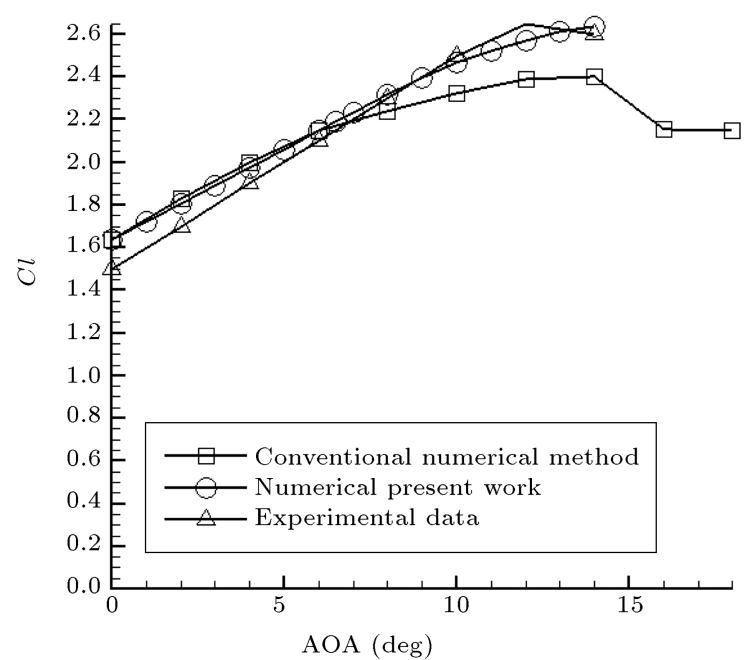

Figure 16. A comparison between the presented method and the conventional numerical method in predicting the lift coefficients.

these small angles of attack, the slot is not practically functional. As the angle of attack increases, the slot performs better and postpones the flow separation. At a specific angle of attack (usually 6-8 degree), the flow separation stops. This is the reason why the CFD code can precisely predict the lift at these moderate angles of attack. Further increasing the angle of attack may again have diverse effects, since leading edge separation and flow instability can occur. Leading edge separation shows itself with a kind of under estimation in the predicted lift results. This phenomenon can be seen in Figure 15.

Another notable point is that severe errors could occur if a conventional numerical approach is taken. Figure 16 compares the two strategies in numerical simulation; one of these strategies is the method discussed in this article, which calculates the lift coefficient for lower angles of attack and estimates the near stall behavior according to semi-empirical methods, and the other is the conventional numerical modeling of flow at all angles of attack. It can be easily observed that the method presented in this article can severely reduce the uncertainty of the calculations, while it does not add more complexity to the numerical solutions.

\section{Conclusion}

A novel method for quick evaluation of the lift producing ability of a high-lift configuration was presented. The main advantages of this method are: quick response, ease of implementation and acceptable accuracy. As explained in this paper, the presented method calculates the linear range of lift curve using computational fluid dynamics, and estimates the near stall region, based on empirical methods. The maximum lift coefficients, estimated using this method, was successfully in consistency with experimental values. It was also shown that a studied unstructured grid can be as good as a structured grid, while other effects, like wind tunnel wall, Re and Mach number, are truly considered. The other important deduction was the fact that low Reynolds turbulence models can generally simulate high lift flow better, although the Spalart-Almaras model is also very good. The presented method can be adequately used in the early phases of aircraft design to reduce costs and improve accuracy.

\section{References}

1. Minervino, M., Lanelli, P. and Quagliarella, D. "3D flap design using Navier-Stokes equations and evolutionary optimization techniques on an industrial platform", Eurogen Conf., CIRA, Capua Italy (2011).

2. Schuster, D.M. and Birckelbaw, L.D. "Numerical computation of viscous flow fields about multiple component airfoils", AIAA Paper 85-0167 (January 1985).

3. Fritz, W. "Calculation of maximum and high lift characteristics of multi element airfoils", High-Lift System Aerodynamics, AGARD CP 515, pp. 5.1-5.12. (September 1993).

4. Godin, P., Zingg, D.W. and Nelson, T.E. "Highlift aerodynamic computations with one- and twoequation turbulence models", A IA A J., 35(2), pp. 23743 (1997); Also AIAA Paper 96-0567 (January 1996).

5. Frhr., H., Geyr, V. and Schade, N. "Prediction of maximum lift effects on realistic high-lift commercialaircraft-configurations within the European project EUROLIFT II", Second Symposium of Simulation of Wing and Nacelle Stall, Braunschweig, Germany (June 22nd-23rd, 2010).

6. Brezillon, J., Dwight, R.P. and Wild, J. "Numerical aerodynamic optimization of 3D high-lift configurations", 26th International Congress of the Aeronautical Science, ICAS (2008).

7. Besnard, E., Schmitz, A., Boscher, E., Garcia, N. and Cebeci, T. "Two-dimensional aircraft high lift system design and optimization", AIAA 98-0123 (1998)

8. Cebeci, T., Besnard, Ch. and Chen, H.H. "An interactive boundary layer method for multi element airfoils", Compute Fluids, 27(5-6), pp. 651-61 (1998).

9. Rumsey, Ch.L. and Ying, S. "Prediction of high lift, review of present CFD capability", Progress in Aerospace Science, 38, pp. 145-180 (2002).

10. Abdol-Hamid, Kh.S., 1st AIAA CFD High Lift Prediction Workshop, NASA Langely Research Center, Chicago, June (2010).

11. Klausmeyer, S.M. and Lin, J.C. "Comparative results from a CFD challenge over a 2D three-element high-lift airfoil", NASA Technical Memorandum (May 1997). 
12. Soltani, M.R., Ebrahimi, A. and Davari, A.R. "A new method for predicting the amplitude and frequency of a highly swept wing undergoing rocking motion", Scientia Iranica, 10(2), pp. 175-185 (2003).

13. Soltani, M.R. and Davari, A.R. "A new approach to investigate unsteady aerodynamic phenomena", Scientia Iranica Journal, 12(4), pp. 379-391 (2005).

14. Roskam, J., Airplane Design, DAR Corporation, Parts 2 and $6(1985)$.

15. Wenzinger, C.J. and Harris, T., Wind Tunnel Investigation of an N.A.C.A. 23012 Airfoil with Various Arrangements of Slotted Flap, National Advisory Comity for Aerodynamic, Report No. 664 (1982).

16. Abbott, I. and Von Doenhoff, A.E., Theory of Wing Sections, Dover Publication Inc., NY (1949).

17. Cao, H.V. and Kusunose, K. "Grid generation and Navier-Stokes analysis for multi-element airfoils", AIAA Paper 94-0748 (January 1994).

18. Rogers, S.E., Roth, K., Nash, S.M., Baker, M.D., Slotnick, J.P., Whitlock, M. and Cao, H.V. "Advances in overset CFD processes applied to subsonic high-lift aircraft", AIAA Paper 2000-4216 (August 2000).
19. Rumyantsev, A.G. and Silantiev, V.A. "Numerical and experimental study of high-lift configurations", Thermo-physics and Aeromechanics, 17(2), pp. 269284 (2010).

\section{Biographies}

Mahmoud Pasandideh Fard is Associate professor of Mechanical and Aerospace Engineering at Ferdowsi University of Mashhad, Iran. He currently teaches more than 10 different courses at the university, mainly in regard to $\mathrm{MS}$ and $\mathrm{PhD}$ degree theses. His research interests are mainly computational low and high speed aerodynamics, boundary layer flow, hydrodynamics and cavitation, and supersonic projectiles.

Seyed Ali Sahaf was born in 1987. He is currently a $\mathrm{PhD}$ degree student in the field of Mechanical Engineering, and is working in an additive manufacturing company. He also worked at the SARI research institute for more than two years in the high lift system design group. His research interests are aircraft design, UAV design and systems integration, aerodynamics, fluid mechanics and plasma flow control. 